Acta Crystallographica Section E

Structure Reports

Online

ISSN 1600-5368

\section{Diaquabis(ethylenediamine)copper(II) bis(4-nitrobenzoate)}

In the title compound, $\left[\mathrm{Cu}\left(\mathrm{C}_{2} \mathrm{H}_{8} \mathrm{~N}_{2}\right)_{2}\left(\mathrm{H}_{2} \mathrm{O}\right)_{2}\right]\left(\mathrm{C}_{7} \mathrm{H}_{4} \mathrm{NO}_{4}\right)_{2}$, the component complex cations and organic anions interact by way of $\mathrm{N}-\mathrm{H} \cdots \mathrm{O}$ and $\mathrm{O}-\mathrm{H} \cdots \mathrm{O}$ hydrogen bonds, leading to a layered structure. The $\mathrm{Cu}$ atom has site symmetry $\overline{1}$.

\section{Comment}

The title compound, (I), was prepared as part of our ongoing studies of second-sphere hydrogen-bonding interactions in compounds containing cationic metal complexes and organic counter-anions (Sharma, Bala et al., 2006; Sharma, Sharma et al., 2006).

Correspondence e-mail: w.harrison@abdn.ac.uk rpsharma@yahoo.co.in

\author{
Key indicators \\ Single-crystal X-ray study \\ $T=93 \mathrm{~K}$ \\ Mean $\sigma(\mathrm{C}-\mathrm{C})=0.004 \AA$ \\ $R$ factor $=0.034$ \\ $w R$ factor $=0.077$ \\ Data-to-parameter ratio $=12.5$
}

For details of how these key indicators were automatically derived from the article, see http://journals.iucr.org/e.
(C) 2007 International Union of Crystallography All rights reserved

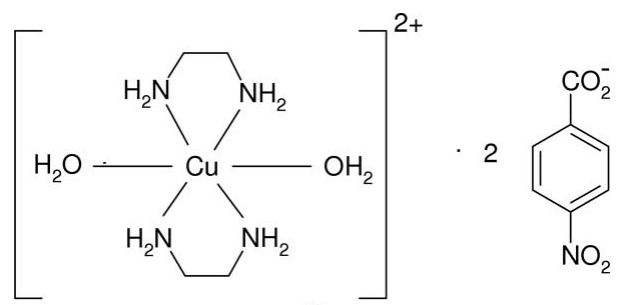

(I)

The geometrical parameters for the component species in (I) fall within their expected ranges (Allen et al., 1987). The well known $\left[\mathrm{Cu}\left(\mathrm{C}_{2} \mathrm{~N}_{2} \mathrm{H}_{8}\right)_{2}\left(\mathrm{H}_{2} \mathrm{O}\right)_{2}\right]^{2+}$ complex cation in (I) is built up from a central copper(II) ion (site symmetry $\overline{1}$ ) chelated by two ethylenediamine molecules to form an approximate $\mathrm{CuN}_{4}$ square. The Jahn-Teller distorted copper coordination is completed by two trans water molecules (Table 1). The $\mathrm{Cu}-\mathrm{N}$ and $\mathrm{Cu}-\mathrm{O}$ bond lengths in (I) are very similar to the equivalent values observed for the same complex cation in its bis(naphthalene-2-sulfonate) (Sharma et al., 2005) and bis(4-fluorobenzoate) (Liu et al., 2004) salts.

The 4-nitrobenzoate anion in (I) is almost planar, the dihedral angles between the mean plane of the C3-C8 benzene ring and the planes of its attached $\mathrm{C} 9 / \mathrm{O} 2 / \mathrm{O} 3$ carboxylate and $\mathrm{N} 3 / \mathrm{O} 4 / \mathrm{O} 5$ nitro groups being 2.14 (17) and $1.9(2)^{\circ}$, respectively. The carboxylate $\mathrm{C}-\mathrm{O}$ bond lengths are almost equal, suggesting charge delocalization.

As well as electrostatic forces, the component species in (I) interact by way of $\mathrm{O}-\mathrm{H} \cdots \mathrm{O}$ and $\mathrm{N}-\mathrm{H} \cdots \mathrm{O}$ hydrogen bonds (Table 2). Firstly, adjacent complex cations are linked into chains propagating along [100] by way of translation-related pairs of $\mathrm{N} 1-\mathrm{H} 1 \cdots \mathrm{O} 1^{\mathrm{i}}$ bonds (see Table 2 for symmetry code). A bridging carboxylate atom $\mathrm{O} 3$ also helps to consolidate the chains (Fig. 2). Then, adjacent cations and anions form a distinctive bridged chain propagating along [010] (Fig. 3), where each carboxylate group in the chain accepts no fewer than four hydrogen bonds from its two adjoining cations.
Received 27 October 2006 Accepted 6 December 2006 


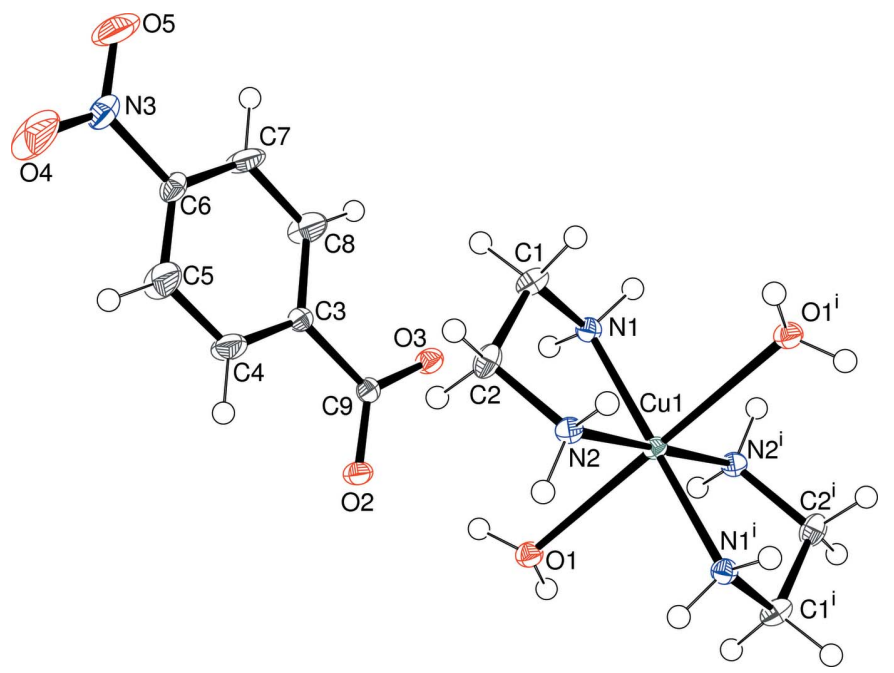

Figure 1

View of the molecular structure of (I), showing $50 \%$ probability displacement ellipsoids (arbitrary spheres for the $\mathrm{H}$ atoms). [Symmetry code: (i) $1-x, 1-y, 1-z$.]

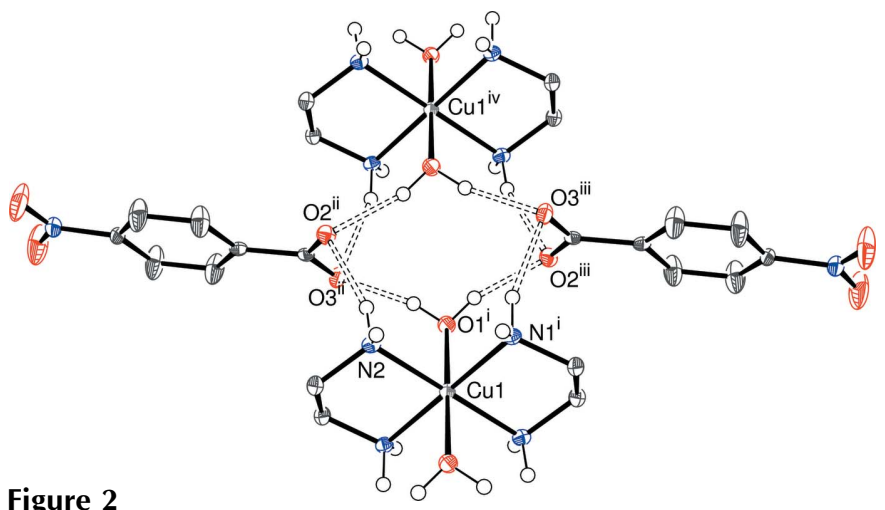

Figure 2

Detail of (I), showing part of a [100] chain arising from hydrogen-bonding interactions (dashed lines). C-bound $\mathrm{H}$ atoms have been omitted. [Symmetry codes: (i) $1-x, 1-y, 1-z$; (ii) $2-x, 1-y, 1-z$; (iii) $1+x$, $y, z ;$ (iv) $1+x, 1+y, z$.]

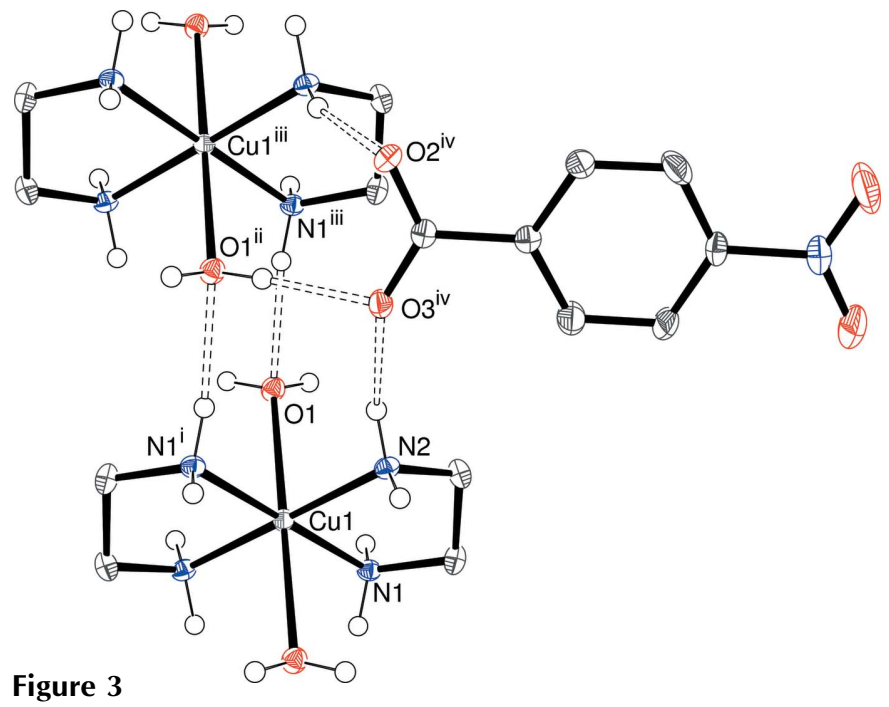

Detail of (I), showing part of a [010] chain arising from hydrogen-bonding interactions (dashed lines). C-bound $\mathrm{H}$ atoms have been omitted. [Symmetry codes: (i) $1-x, 1-y, 1-z$; (ii) $x, 1+y, z$; (iii) $1-x, 2-y$, $1-z ;$ (iv) $x, 1+y, z$.]
Combining these hydrogen-bonding motifs results in (001) sheets of tightly bound cations and anions. It is notable that the nitro $\mathrm{O}$ atoms do not serve as acceptors for any of the hydrogen bonds.

\section{Experimental}

Compound (I) was prepared by taking a suspension of $\left[\mathrm{Cu}\left(\mathrm{H}_{2} \mathrm{O}\right)_{6}\right]\left(\mathrm{C}_{7} \mathrm{H}_{4} \mathrm{NO}_{4}\right)_{2}$ [obtained by reacting basic copper(II) carbonate with $p$-nitrobenzoic acid in water] and adding a methanol solution of ethylenediamine dropwise until a slight excess of a 1:2 Cuen stoichiometry was achieved, resulting in a deep-blue solution, which was allowed to evaporate at room temperature to obtain purple crystals of (I) after a few days. Crystals were filtered off and dried in air.

\section{Crystal data}

$\left[\mathrm{Cu}\left(\mathrm{C}_{2} \mathrm{H}_{8} \mathrm{~N}_{2}\right)_{2}\left(\mathrm{H}_{2} \mathrm{O}\right)_{2}\right]\left(\mathrm{C}_{7} \mathrm{H}_{4} \mathrm{NO}_{4}\right)_{2}$ $M_{r}=552.00$

Triclinic, $P \overline{1}$

$a=6.0019(5) \AA$

$b=7.1230(4) \AA$

$c=15.370(2) \AA$

$\alpha=95.48(2)^{\circ}$

$\beta=98.43(2)^{\circ}$

$\gamma=114.26(2)$

$V=583.69(15) \AA^{3}$

$Z=1$

$D_{x}=1.570 \mathrm{Mg} \mathrm{m}^{-3}$

Mo $K \alpha$ radiation

$\mu=1.00 \mathrm{~mm}^{-1}$

$T=93(2) \mathrm{K}$

Cube, purple

$0.10 \times 0.10 \times 0.10 \mathrm{~mm}$

\section{Data collection}

Rigaku Mercury CCD

diffractometer

$\varphi$ and $\omega$ scans

Absorption correction: multi-scan ( ABSCOR; Higashi, 1995)

$T_{\min }=0.856, T_{\max }=1.000$

(expected range $=0.774-0.905)$

3754 measured reflections 2005 independent reflections 1930 reflections with $I>2 \sigma(I)$ $R_{\text {int }}=0.015$

$\theta_{\max }=25.3^{\circ}$

\section{Refinement}

Refinement on $F^{2}$

$R\left[F^{2}>2 \sigma\left(F^{2}\right)\right]=0.035$

$w R\left(F^{2}\right)=0.077$

$S=1.08$

2005 reflections

160 parameters

$\mathrm{H}$-atom parameters constrained

$$
\begin{gathered}
w=1 /\left[\sigma^{2}\left(F_{\mathrm{o}}^{2}\right)+(0.0337 P)^{2}\right. \\
\quad+0.5044 P] \\
\text { where } P=\left(F_{\mathrm{o}}{ }^{2}+2 F_{\mathrm{c}}^{2}\right) / 3 \\
(\Delta / \sigma)_{\max }=0.002 \\
\Delta \rho_{\max }=0.43 \mathrm{e}^{-3} \\
\Delta \rho_{\min }=-0.44 \mathrm{e}^{-3}
\end{gathered}
$$

Table 1

Selected bond lengths ( $)$.

\begin{tabular}{llll}
\hline $\mathrm{Cu} 1-\mathrm{N} 1$ & $2.0146(18)$ & $\mathrm{C} 9-\mathrm{O} 2$ & $1.256(3)$ \\
$\mathrm{Cu} 1-\mathrm{N} 2$ & $2.0272(19)$ & $\mathrm{C} 9-\mathrm{O} 3$ & $1.261(3)$ \\
$\mathrm{Cu} 1-\mathrm{O} 1$ & $2.5369(17)$ & & \\
\hline
\end{tabular}

Table 2

Hydrogen-bond geometry $\left(\AA,^{\circ}\right)$.

\begin{tabular}{lllll}
\hline$D-\mathrm{H} \cdots A$ & $D-\mathrm{H}$ & $\mathrm{H} \cdots A$ & $D \cdots A$ & $D-\mathrm{H} \cdots A$ \\
\hline $\mathrm{N} 1-\mathrm{H} 1 \cdots \mathrm{O} 1^{\mathrm{i}}$ & 0.92 & 2.11 & $3.018(2)$ & 170 \\
$\mathrm{~N} 1-\mathrm{H} 2 \cdots \mathrm{O} 3$ & 0.92 & 2.20 & $3.081(2)$ & 161 \\
$\mathrm{~N} 2-\mathrm{H} 3 \cdots 3^{\mathrm{ii}}$ & 0.92 & 2.24 & $3.037(3)$ & 145 \\
$\mathrm{~N} 2-\mathrm{H} 4 \cdots$ O2 $^{\mathrm{iii}}$ & 0.92 & 2.07 & $2.960(2)$ & 162 \\
$\mathrm{O}^{\mathrm{i}}-\mathrm{H} 5 \cdots 3^{\mathrm{i}}$ & 0.89 & 1.88 & $2.754(2)$ & 166 \\
$\mathrm{O}^{2}-\mathrm{H} 6 \cdots \mathrm{O} 2$ & 0.86 & 1.93 & $2.767(2)$ & 164 \\
\hline
\end{tabular}

Symmetry codes: (i) $x-1, y, z ; \quad$ (ii) $x+1, y+1, z ; \quad$ (iii) $x, y+1, z ; \quad$ (iv) $-x+1,-y,-z+1$. 


\section{metal-organic papers}

The O-bound $\mathrm{H}$ atoms were located in a difference map and refined as riding in their as-found relative positions with $U_{\text {iso }}(\mathrm{H})=$ $1.2 U_{\text {eq }}(\mathrm{O})$. The $\mathrm{C}$ - and $\mathrm{N}$-bound $\mathrm{H}$ atoms were geometrically placed $(\mathrm{C}-\mathrm{H}=0.95-0.99 \AA, \mathrm{N}-\mathrm{H}=0.92 \AA)$ and refined as riding with $U_{\text {iso }}(\mathrm{H})=1.2 U_{\text {eq }}(\mathrm{C}, \mathrm{N})$.

Data collection: CrystalClear (Rigaku, 2004); cell refinement: CrystalClear; data reduction: CrystalClear; program(s) used to solve structure: SHELXS97 (Sheldrick, 1997); program(s) used to refine structure: SHELXL97 (Sheldrick, 1997); molecular graphics: ORTEP-3 (Farrugia, 1997); software used to prepare material for publication: SHELXL97.

The authors gratefully acknowledge the financial support of UGC vide grant No. F.12-38/2003(SR).

\section{References}

Allen, F. H., Kennard, O., Watson, D., Brammer, L., Orpen, A. G. \& Taylor, R. (1987). J. Chem. Soc. Perkin Trans. 2, pp. S1-19.

Farrugia, L. J. (1997). J. Appl. Cryst. 30, 565.

Higashi, T. (1995). ABSCOR. Rigaku Corporation, Tokyo, Japan.

Liu, Z.-D., Tan, M.-Y. \& Zhu, H.-L. (2004). Acta Cryst. E60, m1081-m1083.

Rigaku (2004). CrystalClear. Rigaku Corporation, Tokyo, Japan.

Sharma, R. P., Bala, R., Sharma, R., Perez, J. \& Miguel, D. (2006). J. Mol. Struct. 797, 49-55.

Sharma, R. P., Sharma, R., Bala, R., Burrows, A. D., Mahon, M. F. \& Cassar, K. (2006). J. Mol. Struct. 794, 173-180.

Sharma, R. P., Sharma, R., Bala, R., Rychlewska, U. \& Warzajtis, B. (2005). J. Mol. Struct. 738, 291-298.

Sheldrick, G. M. (1997). SHELXS97 and SHELXL97. University of Göttingen, Germany. 


\section{supporting information}

Acta Cryst. (2007). E63, m178-m180 [https://doi.org/10.1107/S1600536806052901]

\section{Diaquabis(ethylenediamine)copper(II) bis(4-nitrobenzoate)}

\section{William T. A. Harrison, Alexandra M. Z. Slawin, Raj Pal Sharma, Bhavna Sharma and Shekar Bhama}

Diaquabis(ethylenediamine)copper(II) bis(4-nitrobenzoate)

Crystal data

$\left[\mathrm{Cu}\left(\mathrm{C}_{2} \mathrm{H}_{8} \mathrm{~N}_{2}\right)_{2}\left(\mathrm{H}_{2} \mathrm{O}\right)_{2}\right]\left(\mathrm{C}_{7} \mathrm{H}_{4} \mathrm{NO}_{4}\right)_{2}$

$M_{r}=552.00$

Triclinic, $P \overline{1}$

Hall symbol: -P 1

$a=6.0019(5) \AA$

$b=7.1230(4) \AA$

$c=15.370(2) \AA$

$\alpha=95.48(2)^{\circ}$

$\beta=98.43(2)^{\circ}$

$\gamma=114.26(2)^{\circ}$

$V=583.69(15) \AA^{3}$

Data collection

Rigaku Mercury CCD diffractometer

Radiation source: rotating anode

Confocal monochromator

Detector resolution: 0.83 pixels $\mathrm{mm}^{-1}$

$\varphi$ and $\omega$ scans

Absorption correction: multi-scan

(ABSCOR; Higashi, 1995)

$T_{\text {min }}=0.856, T_{\text {max }}=1.000$

Refinement

Refinement on $F^{2}$

Least-squares matrix: full

$R\left[F^{2}>2 \sigma\left(F^{2}\right)\right]=0.035$

$w R\left(F^{2}\right)=0.077$

$S=1.08$

2005 reflections

160 parameters

0 restraints

Primary atom site location: structure-invariant

direct methods
$Z=1$

$F(000)=287$

$D_{\mathrm{x}}=1.570 \mathrm{Mg} \mathrm{m}^{-3}$

Mo $K \alpha$ radiation, $\lambda=0.71073 \AA$

Cell parameters from 2340 reflections

$\theta=2.7-28.2^{\circ}$

$\mu=1.00 \mathrm{~mm}^{-1}$

$T=93 \mathrm{~K}$

Cube, purple

$0.10 \times 0.10 \times 0.10 \mathrm{~mm}$

3754 measured reflections

2005 independent reflections

1930 reflections with $I>2 \sigma(I)$

$R_{\text {int }}=0.015$

$\theta_{\max }=25.3^{\circ}, \theta_{\min }=2.7^{\circ}$

$h=-5 \rightarrow 7$

$k=-6 \rightarrow 8$

$l=-18 \rightarrow 18$

Secondary atom site location: difference Fourier map

Hydrogen site location: inferred from neighbouring sites

$\mathrm{H}$-atom parameters constrained

$w=1 /\left[\sigma^{2}\left(F_{\mathrm{o}}^{2}\right)+(0.0337 P)^{2}+0.5044 P\right]$

where $P=\left(F_{\mathrm{o}}{ }^{2}+2 F_{\mathrm{c}}{ }^{2}\right) / 3$

$(\Delta / \sigma)_{\max }=0.002$

$\Delta \rho_{\max }=0.43 \mathrm{e} \AA^{-3}$

$\Delta \rho_{\min }=-0.44$ e $\AA^{-3}$ 


\section{Special details}

Geometry. All e.s.d.'s (except the e.s.d. in the dihedral angle between two 1.s. planes) are estimated using the full covariance matrix. The cell e.s.d.'s are taken into account individually in the estimation of e.s.d.'s in distances, angles and torsion angles; correlations between e.s.d.'s in cell parameters are only used when they are defined by crystal symmetry. An approximate (isotropic) treatment of cell e.s.d.'s is used for estimating e.s.d.'s involving l.s. planes.

Refinement. Refinement of $F^{2}$ against ALL reflections. The weighted $R$-factor $w R$ and goodness of fit $S$ are based on $F^{2}$, conventional $R$-factors $R$ are based on $F$, with $F$ set to zero for negative $F^{2}$. The threshold expression of $F^{2}>\sigma\left(F^{2}\right)$ is used only for calculating $R$-factors (gt) etc. and is not relevant to the choice of reflections for refinement. $R$-factors based on $F^{2}$ are statistically about twice as large as those based on $F$, and $R$ - factors based on ALL data will be even larger.

Fractional atomic coordinates and isotropic or equivalent isotropic displacement parameters $\left(\hat{A}^{2}\right)$

\begin{tabular}{lllll}
\hline & $x$ & $y$ & $z$ & $U_{\text {iso }} * / U_{\text {eq }}$ \\
\hline Cu1 & 0.5000 & 0.5000 & 0.5000 & $0.01301(14)$ \\
C1 & $0.3527(4)$ & $0.4877(4)$ & $0.66944(15)$ & $0.0177(5)$ \\
H1A & 0.2872 & 0.4000 & 0.7143 & $0.021^{*}$ \\
H1B & 0.2922 & 0.5978 & 0.6710 & $0.021^{*}$ \\
C2 & $0.6352(4)$ & $0.5864(4)$ & $0.69053(15)$ & $0.0177(5)$ \\
H2A & 0.6998 & 0.6899 & 0.7465 & $0.021^{*}$ \\
H2B & 0.6960 & 0.4780 & 0.6983 & $0.021^{*}$ \\
N1 & $0.2647(3)$ & $0.3572(3)$ & $0.57892(12)$ & $0.0131(4)$ \\
H1 & 0.1061 & 0.3396 & 0.5551 & $0.016^{*}$ \\
H2 & 0.2599 & 0.2274 & 0.5826 & $0.016^{*}$ \\
N2 & $0.7230(3)$ & $0.6905(3)$ & $0.61493(12)$ & $0.0138(4)$ \\
H3 & 0.8861 & 0.7141 & 0.6160 & $0.017^{*}$ \\
H4 & 0.7145 & 0.8170 & 0.6191 & $0.017^{*}$ \\
O1 & $0.7238(3)$ & $0.2674(2)$ & $0.51837(10)$ & $0.0158(4)$ \\
H5 & 0.7064 & 0.1756 & 0.4710 & $0.019^{*}$ \\
H6 & 0.6952 & 0.1896 & 0.5589 & $0.019^{*}$ \\
C3 & $0.4867(4)$ & $0.1037(3)$ & $0.78913(14)$ & $0.0139(5)$ \\
C4 & $0.7093(5)$ & $0.2037(5)$ & $0.85044(17)$ & $0.0297(6)$ \\
H4A & 0.8620 & 0.2341 & 0.8313 & $0.036^{*}$ \\
C5 & $0.7131(5)$ & $0.2603(5)$ & $0.93950(17)$ & $0.0354(7)$ \\
H5A & 0.8666 & 0.3270 & 0.9818 & $0.042^{*}$ \\
C6 & $0.4916(4)$ & $0.2183(3)$ & $0.96539(15)$ & $0.0167(5)$ \\
C7 & $0.2672(5)$ & $0.1185(5)$ & $0.90654(17)$ & $0.0350(7)$ \\
H7 & 0.1152 & 0.0891 & 0.9262 & $0.042^{*}$ \\
C8 & $0.2663(5)$ & $0.0614(5)$ & $0.81761(17)$ & $0.0330(7)$ \\
H8 & 0.1120 & -0.0078 & 0.7759 & $0.040^{*}$ \\
C9 & $0.4873(4)$ & $0.0454(3)$ & $0.69155(14)$ & $0.0136(5)$ \\
N3 & $0.4942(4)$ & $0.2855(3)$ & $1.05959(13)$ & $0.0222(5)$ \\
O2 & $0.6956(3)$ & $0.0885(2)$ & $0.67087(10)$ & $0.0171(4)$ \\
O3 & $0.2795(3)$ & $-0.0400(2)$ & $0.63757(10)$ & $0.0183(4)$ \\
O4 & $0.6929(4)$ & $0.3750(4)$ & $1.11118(13)$ & $0.0516(6)$ \\
O5 & $0.2963(4)$ & $0.2486(4)$ & $1.08147(13)$ & $0.0499(6)$ \\
& & & &
\end{tabular}


Atomic displacement parameters $\left(\AA^{2}\right)$

\begin{tabular}{lllllll}
\hline & $U^{11}$ & $U^{22}$ & $U^{33}$ & $U^{12}$ & $U^{13}$ & $U^{23}$ \\
\hline $\mathrm{Cu} 1$ & $0.0109(2)$ & $0.0139(2)$ & $0.0121(2)$ & $0.00344(16)$ & $0.00238(15)$ & $0.00153(15)$ \\
$\mathrm{C} 1$ & $0.0215(13)$ & $0.0168(13)$ & $0.0140(11)$ & $0.0066(10)$ & $0.0070(10)$ & $0.0020(9)$ \\
$\mathrm{C} 2$ & $0.0204(13)$ & $0.0176(13)$ & $0.0124(11)$ & $0.0065(10)$ & $0.0012(10)$ & $0.0017(9)$ \\
$\mathrm{N} 1$ & $0.0124(9)$ & $0.0120(10)$ & $0.0152(9)$ & $0.0059(8)$ & $0.0019(8)$ & $0.0018(7)$ \\
$\mathrm{N} 2$ & $0.0111(9)$ & $0.0127(10)$ & $0.0172(10)$ & $0.0048(8)$ & $0.0037(8)$ & $0.0017(8)$ \\
O1 & $0.0175(8)$ & $0.0155(8)$ & $0.0158(8)$ & $0.0080(7)$ & $0.0046(7)$ & $0.0032(6)$ \\
$\mathrm{C} 3$ & $0.0172(12)$ & $0.0093(12)$ & $0.0164(11)$ & $0.0067(9)$ & $0.0034(9)$ & $0.0025(9)$ \\
$\mathrm{C} 4$ & $0.0155(13)$ & $0.0513(18)$ & $0.0181(13)$ & $0.0107(12)$ & $0.0046(10)$ & $0.0028(12)$ \\
$\mathrm{C} 5$ & $0.0165(14)$ & $0.0567(19)$ & $0.0168(13)$ & $0.0039(13)$ & $-0.0026(11)$ & $-0.0018(12)$ \\
$\mathrm{C} 6$ & $0.0261(13)$ & $0.0135(12)$ & $0.0118(11)$ & $0.0096(10)$ & $0.0050(10)$ & $0.0015(9)$ \\
$\mathrm{C} 7$ & $0.0181(14)$ & $0.063(2)$ & $0.0184(13)$ & $0.0124(13)$ & $0.0080(11)$ & $-0.0036(13)$ \\
$\mathrm{C} 8$ & $0.0153(13)$ & $0.0508(18)$ & $0.0197(13)$ & $0.0038(12)$ & $0.0034(11)$ & $-0.0050(12)$ \\
$\mathrm{C} 9$ & $0.0191(12)$ & $0.0086(11)$ & $0.0152(11)$ & $0.0073(9)$ & $0.0047(10)$ & $0.0037(9)$ \\
$\mathrm{N} 3$ & $0.0327(13)$ & $0.0195(11)$ & $0.0138(10)$ & $0.0106(10)$ & $0.0052(10)$ & $0.0020(8)$ \\
O2 & $0.0167(8)$ & $0.0188(9)$ & $0.0183(8)$ & $0.0086(7)$ & $0.0075(7)$ & $0.0038(7)$ \\
O3 & $0.0162(9)$ & $0.0215(9)$ & $0.0135(8)$ & $0.0054(7)$ & $0.0027(7)$ & $-0.0005(6)$ \\
O4 & $0.0385(13)$ & $0.0721(16)$ & $0.0171(10)$ & $0.0033(11)$ & $0.0003(10)$ & $-0.0118(10)$ \\
O5 & $0.0417(13)$ & $0.0851(18)$ & $0.0219(11)$ & $0.0273(12)$ & $0.0131(10)$ & $-0.0052(11)$ \\
& & & & & & \\
\hline
\end{tabular}

Geometric parameters $\left(\AA,{ }^{\circ}\right)$

\begin{tabular}{llll}
\hline $\mathrm{Cu} 1-\mathrm{N} 1^{\mathrm{i}}$ & $2.0146(18)$ & $\mathrm{O} 1-\mathrm{H} 6$ & 0.8625 \\
$\mathrm{Cu} 1-\mathrm{N} 1$ & $2.0146(18)$ & $\mathrm{C} 3-\mathrm{C} 8$ & $1.378(3)$ \\
$\mathrm{Cu} 1-\mathrm{N} 2$ & $2.0272(19)$ & $\mathrm{C} 3-\mathrm{C} 4$ & $1.379(3)$ \\
$\mathrm{Cu} 1-\mathrm{N} 2^{\mathrm{i}}$ & $2.0272(19)$ & $\mathrm{C} 3-\mathrm{C} 9$ & $1.519(3)$ \\
$\mathrm{Cu} 1-\mathrm{O} 1$ & $2.5369(17)$ & $\mathrm{C} 4-\mathrm{C} 5$ & $1.384(4)$ \\
$\mathrm{Cu} 1-\mathrm{O} 1^{\mathrm{i}}$ & $2.5369(17)$ & $\mathrm{C} 4-\mathrm{H} 4 \mathrm{~A}$ & 0.950 \\
$\mathrm{C} 1-\mathrm{N} 1$ & $1.488(3)$ & $\mathrm{C} 5-\mathrm{C} 6$ & $1.366(4)$ \\
$\mathrm{C} 1-\mathrm{C} 2$ & $1.513(3)$ & $\mathrm{C} 5-\mathrm{H} 5 \mathrm{~A}$ & 0.950 \\
$\mathrm{C} 1-\mathrm{H} 1 \mathrm{~A}$ & 0.990 & $\mathrm{C} 6-\mathrm{C} 7$ & $1.368(4)$ \\
$\mathrm{C} 1-\mathrm{H} 1 \mathrm{~B}$ & 0.990 & $\mathrm{C} 6-\mathrm{N} 3$ & $1.477(3)$ \\
$\mathrm{C} 2-\mathrm{N} 2$ & $1.483(3)$ & $\mathrm{C} 7-\mathrm{C} 8$ & $1.387(4)$ \\
$\mathrm{C} 2-\mathrm{H} 2 \mathrm{~A}$ & 0.990 & $\mathrm{C} 7-\mathrm{H} 7$ & 0.950 \\
$\mathrm{C} 2-\mathrm{H} 2 \mathrm{~B}$ & 0.990 & $\mathrm{C} 8-\mathrm{H} 8$ & 0.950 \\
$\mathrm{~N} 1-\mathrm{H} 1$ & 0.920 & $\mathrm{C} 9-\mathrm{O} 2$ & $1.256(3)$ \\
$\mathrm{N} 1-\mathrm{H} 2$ & 0.920 & $\mathrm{C} 9-\mathrm{O} 3$ & $1.261(3)$ \\
$\mathrm{N} 2-\mathrm{H} 3$ & 0.920 & $\mathrm{~N} 3-\mathrm{O} 4$ & $1.208(3)$ \\
$\mathrm{N} 2-\mathrm{H} 4$ & 0.920 & $\mathrm{~N} 3-\mathrm{O} 5$ & $1.212(3)$ \\
$\mathrm{O} 1-\mathrm{H} 5$ & 0.8941 & & 108.3 \\
& & & $107.93(14)$ \\
$\mathrm{N} 1-\mathrm{Cu} 1-\mathrm{N} 1$ & 180.0 & $\mathrm{H} 1-\mathrm{N} 1-\mathrm{H} 2$ & 110.1 \\
$\mathrm{~N} 1-\mathrm{Cu} 1-\mathrm{N} 2$ & $95.08(7)$ & $\mathrm{C} 2-\mathrm{N} 2-\mathrm{N} 2-\mathrm{H} 3$ & 110.1 \\
$\mathrm{~N} 1-\mathrm{Cu} 1-\mathrm{N} 2$ & $84.92(7)$ & $\mathrm{Cu} 1-\mathrm{N} 2-\mathrm{H} 3$ & 110.1 \\
$\mathrm{~N} 1-\mathrm{Cu} 1-\mathrm{N} 2{ }^{\mathrm{i}}$ & $84.92(7)$ & & \\
$\mathrm{N} 1-\mathrm{Cu} 1-\mathrm{N} 22^{\mathrm{i}}$ & $95.08(7)$ & &
\end{tabular}




\begin{tabular}{|c|c|c|c|}
\hline $\mathrm{N} 2-\mathrm{Cu} 1-\mathrm{N} 2^{\mathrm{i}}$ & 180.0 & $\mathrm{Cu} 1-\mathrm{N} 2-\mathrm{H} 4$ & 110.1 \\
\hline $\mathrm{O} 1-\mathrm{Cu} 1-\mathrm{N} 1$ & $92.58(7)$ & $\mathrm{H} 3-\mathrm{N} 2-\mathrm{H} 4$ & 108.4 \\
\hline $\mathrm{O} 1-\mathrm{Cu} 1-\mathrm{N} 2$ & $89.44(7)$ & $\mathrm{H} 5-\mathrm{O} 1-\mathrm{H} 6$ & 101.3 \\
\hline $\mathrm{O} 1-\mathrm{Cu} 1-\mathrm{N} 1^{\mathrm{i}}$ & $87.42(7)$ & $\mathrm{C} 8-\mathrm{C} 3-\mathrm{C} 4$ & $119.0(2)$ \\
\hline $\mathrm{O} 1-\mathrm{Cu} 1-\mathrm{N} 2^{\mathrm{i}}$ & $90.56(7)$ & $\mathrm{C} 8-\mathrm{C} 3-\mathrm{C} 9$ & $121.0(2)$ \\
\hline $\mathrm{O} 1^{\mathrm{i}}-\mathrm{Cu} 1-\mathrm{N} 1^{\mathrm{i}}$ & $92.58(7)$ & $\mathrm{C} 4-\mathrm{C} 3-\mathrm{C} 9$ & $119.9(2)$ \\
\hline $\mathrm{O} 1^{\mathrm{i}}-\mathrm{Cu} 1-\mathrm{N} 2^{\mathrm{i}}$ & $89.44(7)$ & $\mathrm{C} 3-\mathrm{C} 4-\mathrm{C} 5$ & $120.9(2)$ \\
\hline $\mathrm{O} 1{ }^{\mathrm{i}}-\mathrm{Cu} 1-\mathrm{N} 1$ & $87.42(7)$ & $\mathrm{C} 3-\mathrm{C} 4-\mathrm{H} 4 \mathrm{~A}$ & 119.6 \\
\hline $\mathrm{O} 1-\mathrm{Cu} 1-\mathrm{N} 2$ & $90.56(7)$ & $\mathrm{C} 5-\mathrm{C} 4-\mathrm{H} 4 \mathrm{~A}$ & 119.6 \\
\hline $\mathrm{O} 1-\mathrm{Cu} 1-\mathrm{O} 1^{\mathrm{i}}$ & 180.0 & $\mathrm{C} 6-\mathrm{C} 5-\mathrm{C} 4$ & $118.6(2)$ \\
\hline $\mathrm{N} 1-\mathrm{C} 1-\mathrm{C} 2$ & $108.30(18)$ & $\mathrm{C} 6-\mathrm{C} 5-\mathrm{H} 5 \mathrm{~A}$ & 120.7 \\
\hline $\mathrm{N} 1-\mathrm{C} 1-\mathrm{H} 1 \mathrm{~A}$ & 110.0 & $\mathrm{C} 4-\mathrm{C} 5-\mathrm{H} 5 \mathrm{~A}$ & 120.7 \\
\hline $\mathrm{C} 2-\mathrm{C} 1-\mathrm{H} 1 \mathrm{~A}$ & 110.0 & $\mathrm{C} 5-\mathrm{C} 6-\mathrm{C} 7$ & $122.1(2)$ \\
\hline $\mathrm{N} 1-\mathrm{C} 1-\mathrm{H} 1 \mathrm{~B}$ & 110.0 & $\mathrm{C} 5-\mathrm{C} 6-\mathrm{N} 3$ & $119.1(2)$ \\
\hline $\mathrm{C} 2-\mathrm{C} 1-\mathrm{H} 1 \mathrm{~B}$ & 110.0 & $\mathrm{C} 7-\mathrm{C} 6-\mathrm{N} 3$ & $118.8(2)$ \\
\hline $\mathrm{H} 1 \mathrm{~A}-\mathrm{C} 1-\mathrm{H} 1 \mathrm{~B}$ & 108.4 & $\mathrm{C} 6-\mathrm{C} 7-\mathrm{C} 8$ & $118.6(2)$ \\
\hline $\mathrm{N} 2-\mathrm{C} 2-\mathrm{C} 1$ & $107.83(18)$ & $\mathrm{C} 6-\mathrm{C} 7-\mathrm{H} 7$ & 120.7 \\
\hline $\mathrm{N} 2-\mathrm{C} 2-\mathrm{H} 2 \mathrm{~A}$ & 110.1 & $\mathrm{C} 8-\mathrm{C} 7-\mathrm{H} 7$ & 120.7 \\
\hline $\mathrm{C} 1-\mathrm{C} 2-\mathrm{H} 2 \mathrm{~A}$ & 110.1 & $\mathrm{C} 3-\mathrm{C} 8-\mathrm{C} 7$ & $120.8(2)$ \\
\hline $\mathrm{N} 2-\mathrm{C} 2-\mathrm{H} 2 \mathrm{~B}$ & 110.1 & $\mathrm{C} 3-\mathrm{C} 8-\mathrm{H} 8$ & 119.6 \\
\hline $\mathrm{C} 1-\mathrm{C} 2-\mathrm{H} 2 \mathrm{~B}$ & 110.1 & $\mathrm{C} 7-\mathrm{C} 8-\mathrm{H} 8$ & 119.6 \\
\hline $\mathrm{H} 2 \mathrm{~A}-\mathrm{C} 2-\mathrm{H} 2 \mathrm{~B}$ & 108.5 & $\mathrm{O} 2-\mathrm{C} 9-\mathrm{O} 3$ & $125.2(2)$ \\
\hline $\mathrm{C} 1-\mathrm{N} 1-\mathrm{Cu} 1$ & $109.03(13)$ & $\mathrm{O} 2-\mathrm{C} 9-\mathrm{C} 3$ & $117.2(2)$ \\
\hline $\mathrm{C} 1-\mathrm{N} 1-\mathrm{H} 1$ & 109.9 & $\mathrm{O} 3-\mathrm{C} 9-\mathrm{C} 3$ & $117.57(19)$ \\
\hline $\mathrm{Cu} 1-\mathrm{N} 1-\mathrm{H} 1$ & 109.9 & $\mathrm{O} 4-\mathrm{N} 3-\mathrm{O} 5$ & $123.0(2)$ \\
\hline $\mathrm{C} 1-\mathrm{N} 1-\mathrm{H} 2$ & 109.9 & $\mathrm{O} 4-\mathrm{N} 3-\mathrm{C} 6$ & $118.6(2)$ \\
\hline $\mathrm{Cu} 1-\mathrm{N} 1-\mathrm{H} 2$ & 109.9 & $\mathrm{O} 5-\mathrm{N} 3-\mathrm{C} 6$ & $118.4(2)$ \\
\hline $\mathrm{N} 1-\mathrm{C} 1-\mathrm{C} 2-\mathrm{N} 2$ & $51.7(2)$ & $\mathrm{N} 3-\mathrm{C} 6-\mathrm{C} 7-\mathrm{C} 8$ & $178.1(2)$ \\
\hline $\mathrm{C} 2-\mathrm{C} 1-\mathrm{N} 1-\mathrm{Cu} 1$ & $-36.6(2)$ & $\mathrm{C} 4-\mathrm{C} 3-\mathrm{C} 8-\mathrm{C} 7$ & $0.2(4)$ \\
\hline $\mathrm{N} 2-\mathrm{Cu} 1-\mathrm{N} 1-\mathrm{C} 1$ & $11.08(14)$ & $\mathrm{C} 9-\mathrm{C} 3-\mathrm{C} 8-\mathrm{C} 7$ & $-178.8(3)$ \\
\hline $\mathrm{N} 22^{\mathrm{i}}-\mathrm{Cu} 1-\mathrm{N} 1-\mathrm{C} 1$ & $-168.92(14)$ & $\mathrm{C} 6-\mathrm{C} 7-\mathrm{C} 8-\mathrm{C} 3$ & $0.1(5)$ \\
\hline $\mathrm{C} 1-\mathrm{C} 2-\mathrm{N} 2-\mathrm{Cu} 1$ & $-41.2(2)$ & $\mathrm{C} 8-\mathrm{C} 3-\mathrm{C} 9-\mathrm{O} 2$ & $-179.8(2)$ \\
\hline $\mathrm{N} 1-\mathrm{Cu} 1-\mathrm{N} 2-\mathrm{C} 2$ & $-163.03(14)$ & $\mathrm{C} 4-\mathrm{C} 3-\mathrm{C} 9-\mathrm{O} 2$ & $1.3(3)$ \\
\hline $\mathrm{N} 1-\mathrm{Cu} 1-\mathrm{N} 2-\mathrm{C} 2$ & $16.97(14)$ & $\mathrm{C} 8-\mathrm{C} 3-\mathrm{C} 9-\mathrm{O} 3$ & $1.1(3)$ \\
\hline $\mathrm{C} 8-\mathrm{C} 3-\mathrm{C} 4-\mathrm{C} 5$ & $0.3(4)$ & $\mathrm{C} 4-\mathrm{C} 3-\mathrm{C} 9-\mathrm{O} 3$ & $-177.8(2)$ \\
\hline $\mathrm{C} 9-\mathrm{C} 3-\mathrm{C} 4-\mathrm{C} 5$ & $179.3(2)$ & $\mathrm{C} 5-\mathrm{C} 6-\mathrm{N} 3-\mathrm{O} 4$ & $-0.3(4)$ \\
\hline $\mathrm{C} 3-\mathrm{C} 4-\mathrm{C} 5-\mathrm{C} 6$ & $-1.1(4)$ & $\mathrm{C} 7-\mathrm{C} 6-\mathrm{N} 3-\mathrm{O} 4$ & $-179.4(3)$ \\
\hline $\mathrm{C} 4-\mathrm{C} 5-\mathrm{C} 6-\mathrm{C} 7$ & $1.4(4)$ & $\mathrm{C} 5-\mathrm{C} 6-\mathrm{N} 3-\mathrm{O} 5$ & $179.4(3)$ \\
\hline $\mathrm{C} 4-\mathrm{C} 5-\mathrm{C} 6-\mathrm{N} 3$ & $-177.6(2)$ & $\mathrm{C} 7-\mathrm{C} 6-\mathrm{N} 3-\mathrm{O} 5$ & $0.4(4)$ \\
\hline $\mathrm{C} 5-\mathrm{C} 6-\mathrm{C} 7-\mathrm{C} 8$ & $-0.9(4)$ & & \\
\hline
\end{tabular}

Symmetry code: (i) $-x+1,-y+1,-z+1$.

Hydrogen-bond geometry $\left(\AA,{ }^{\circ}\right)$

\begin{tabular}{lllll}
\hline$D-\mathrm{H} \cdots A$ & $D-\mathrm{H}$ & $\mathrm{H} \cdots A$ & $D \cdots A$ & $D-\mathrm{H} \cdots A$ \\
\hline $\mathrm{N} 1-\mathrm{H} 1 \cdots \mathrm{O} 1^{\mathrm{ii}}$ & 0.92 & 2.11 & $3.018(2)$ & 170
\end{tabular}




$\begin{array}{lllll}\mathrm{N} 1-\mathrm{H} 2 \cdots \mathrm{O} 3 & 0.92 & 2.20 & 3.081(2) & 161 \\ \mathrm{~N} 2-\mathrm{H} 3 \cdots \mathrm{O} 3^{\text {iii }} & 0.92 & 2.24 & 3.037(3) & 145 \\ \mathrm{~N} 2-\mathrm{H} 4 \cdots \mathrm{O} 2^{\text {iv }} & 0.92 & 2.07 & 2.960(2) & 162 \\ \mathrm{O} 1-\mathrm{H} 5 \cdots \mathrm{O} 3^{\mathrm{v}} & 0.89 & 1.88 & 2.754(2) & 166 \\ \mathrm{O} 1-\mathrm{H} 6 \cdots \mathrm{O} 2 & 0.86 & 1.93 & 2.767(2) & 164\end{array}$

Symmetry codes: (ii) $x-1, y, z$; (iii) $x+1, y+1, z$; (iv) $x, y+1, z$; (v) $-x+1,-y,-z+1$. 